\title{
45. Jahrestagung der Rheinisch-Westfälischen Gesellschaft für Nuklearmedizin (RWGN)
}

Am 30. November und 1. Dezember 2018 fand unter der Leitung des Tagungspräsidenten Prof. Dr. Rolf Larisch die 45. Jahrestagung der Rheinisch-Westfälischen Gesellschaft für Nuklearmedizin (RWGN) am Klinikum Lüdenscheid - Märkische Kliniken GmbH in Lüdenscheid statt. Es kamen insgesamt 118 Teilnehmer - davon 33 MTRAs. 21 Referenten boten ein breites Spektrum von wissenschaftlichen und Fortbildungsvorträgen an. Auf der begleitenden, sehr informativen Industrieausstellung waren insgesamt 22 Aussteller vertreten. Während der Tagungspausen und beim gemütlichen Beisammensein war Raum für viele informative und unterhaltsame Gespräche unter den Teilnehmern.

Am ersten Tag der Jahrestagung erfolgte die Präsentationen der aktuellen Arbeit der Universitätsklinika aus NRW. Die Themen erstreckten sich von der Anwendung von PSMA in Diagnostik und Therapie über neuroendokrine Tumore, dem M. Basedow bis hin zu modernen Standards und Randbedingungen der Radiopharmaka-Herstellung. Zum Abschluss des Tages erfolgte die Verleihung des Hans-Creutzig Preises vom Vorsitzenden der RWGN, Herr Prof. Dr. Dr. Wolfgang Schäfer, an den diesjährigen Preisträger Prof. Dr. Frederik L. Giesel aus der Abteilung für Nuklearmedizin, Radiologische Klinik des Universitätsklinikums Heidelberg für die Arbeit „FAPI-PET/CT: biodistribution and preliminary dosimetry estimate of two DOTA-containing FAP-targeting agents in patients with various cancers " publiziert im Journal of Nuclear Medicine [1].
Am zweiten Tag der Veranstaltung wurden praktisch wichtige Themen unter dem Leitgedanken „Grenzen des Normalen, Anfänge des Pathologischen “ referiert. Dies erstreckte sich über klinisch häufige Themen wie die Nierenszintigrafie, die Hirnbildgebung, die Skelettszintigrafie und die Leberszintigrafie. Nach einer Pause schlossen sich die PET-Bildgebung, die Schilddrüsenszintigrafie, die Lungenszintigrafie und die Myokardszintigrafie an.

Parallel hierzu gab es eine Fortbildungsveranstaltung für MTRA zu den Themen Schilddrüsenszintigrafie, Therapie von Schilddrüsenknoten, die Arbeit der ärztlichen Stelle, die Skelettszintigrafie und die Sentinel-Lymphknotenszintigrafie.

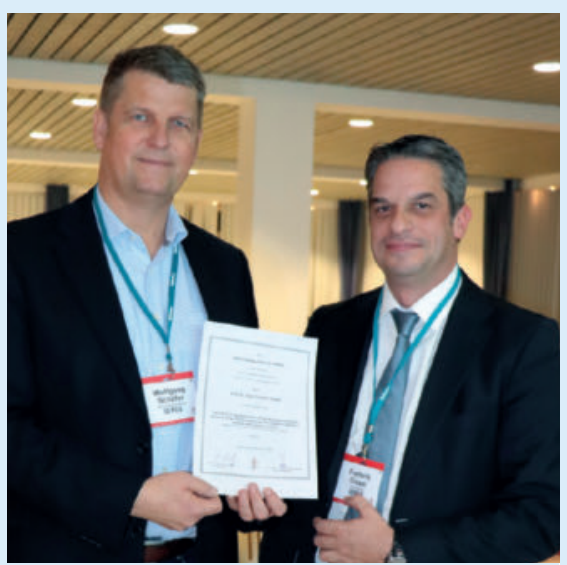

Der Hans-Creutzig-Preisträger Prof. Dr. Frederik. L. Giesel mit dem Vorsitzenden der RWGN, Prof. Dr. Dr. Wolfgang Schäfer (Quelle: Märkische Kliniken GmbH, Klinikum Lüdenscheid).
Herzlichen Dank an alle Teilnehmer und Referenten, an die Organisatoren, an die Industrie für die Unterstützung und den Mitarbeiterinnen der vokativ $\mathrm{GmbH}$ für eine perfekte Organisation.

Die 46. Jahrestagung der RWGN wird am 29. und 30. November 2019 in Bonn unter der Präsidentschaft von Prof. Dr. Holger Palmedo stattfinden.

Prof. Dr. Wolfgang Burchert, Bad Oeynhausen

Literatur

[1] Giesel F, Kratochwil C, Lindner T, Marschalek M, Loktev A, Lehnert W, Debus J, Jäger D, Flechsig P, Altmann A, Mier W, Haberkorn U. FAPI-PET/CT: biodistribution and preliminary dosimetry estimate of two DOTA-containing FAP-targeting agents in patients with various cancers. J Nucl Med 2018; DOI: 10.2967| jnumed.118.215913.

\section{VERANTWORTLICH FÜR DEN} INHALT

Deutsche Gesellschaft für Nuklearmedizin e.V.(DGN)

Prof. Dr. Bernd Joachim Krause

(Präsident)

Nikolaistr. 29, 37073 Göttingen

E-Mail: office@nuklearmedizin.de

Schriftführer:

Prof. Dr. Peter Bartenstein

E-Mail: office@nuklearmedizin.de 